\title{
Review of: "The genome of the endangered Macadamia jansenii displays little diversity but represents an 1 important genetic resource for plant breeding"
}

\section{Zhiguo Dong}

Potential competing interests: The author(s) declared that no potential competing interests exist.

In this manuscript, authors reported a a chromosome level genome assembly of Macadamia jansenii, but the data analysis is very simple. Here, there are some questions that need to be explained.

1.Please ensure the "the N50 of 58 Mb" is contig N50 or scaffold N50? If it is scaffold N50, what is the value of contig N50?

2. the authors mentioned "provides a platform for analysis of three unique attributes of macadamia, the high levels of unusual fatty acids, high cyanogenic glucoside content, and the presence of a novel anti-microbial peptide." Whether to screen related pathways in the article $\square$ What are they $\square$

Did the authors perform relevant experimental verifications on some of the genes they selected in this mauscript? Such as anti-microbial genes, Cyanogenic glycoside genes, or Fatty acid metabolism genes? 